Conferencias, seminarios y trabajos de Matemática

ISSN: 1515.4904

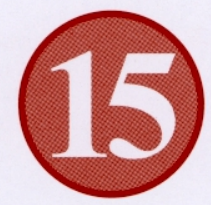

Workshop on

Mathematical

Modelling of Energy

and Mass Transfer

Processes, and

Applications

Domingo A. Tarzia

Rodolfo H. Mascheroni (Eds.) 


\title{
THE CLASSICAL ONE-PHASE STEFAN PROBLEM WITH TEMPERATURE-DEPENDENT THERMAL CONDUCTIVITY AND A CONVECTIVE TERM
}

\author{
M.F. NATALE ${ }^{(1)}$ - D.A. TARZIA ${ }^{(1)(2) *}$
}

(1) Depto. de Matemática , F.C.E.,Universidad Austral, Paraguay 1950, S2000FZF Rosario, Argentina.

(2) CONICET, Argentina

E-mails: Maria.Natale@fce.austral.edu.ar;

Domingo.Tarzia@fce.austral.edu.ar

\begin{abstract}
We study a one-phase Stefan problem for a semi-infinite material with temperaturedependent thermal conductivity and a convective term with a constant temperature boundary condition or a heat flux boundary condition of the type $-q_{0} / \sqrt{t}\left(q_{0}>0\right)$ at the fixed face $x=0$. We obtain in both cases sufficient conditions for data in order to have a parametric representation of the solution of the similarity type for $t \geq t_{0}>0$ with $t_{0}$ an arbitrary positive time. We improve the results given in Rogers-Broadbridge, ZAMP, 39 (1988), 122-129 and Natale-Tarzia, Int. J. Eng. Sci., 41 (2003), 1685-1698 obtaining explicit solutions through the unique solution of a Cauchy problem with the time as a parameter and we also give an algorithm in order to compute the explicit solutions.
\end{abstract}

Key words : Stefan problem, free boundary problem, moving boundary problem, phase-change process, nonlinear thermal conductivity, fusion, solidification, similarity solution.

2000 AMS Subject Classification: 35R35, 80A22, 35C05

\section{Introduction.}

We consider Stefan problems for a semi-infinite region $x>0$ with temperaturedependent thermal conductivity and a convective term with phase change temperature $\theta_{f}=0$ [16]. In all of them is required to determine the evolution of the moving phase separation $x=s(t)$ and the temperature distribution $\theta(x, t)$. The modeling of this kind of systems is a problem with a great mathematical and industrial significance. Phase-change

${ }^{*}$ MAT - Serie A, 15 (2008), 1-16. 
problems appear frequently in industrial processes an other problems of technological interest [1],[2],[8] - [14],[17]. A large bibliography on the subject was given in [27]. We consider one-phase Stefan problems in fusion process with nonlinear heat conduction equations.

Owing to [19], [24] we consider the following free boundary (fusion process) problem

$$
\begin{gathered}
\rho c \frac{\partial \theta}{\partial t}=\frac{\partial}{\partial x}\left(k(\theta, x) \frac{\partial \theta}{\partial x}\right)-v(\theta) \frac{\partial \theta}{\partial x}, 0<x<s(t), t>0 \\
k(\theta(0, t), 0) \frac{\partial \theta}{\partial x}(0, t)=-\frac{q_{0}}{\sqrt{t}}, q_{0}>0, t>0 \\
k(\theta(s(t), t), s(t)) \frac{\partial \theta}{\partial x}(s(t), t)=-\rho l \dot{s}(t), t>0 \\
\theta(s(t), t)=0, t>0, s(0)=0
\end{gathered}
$$

where the thermal conductivity $k(\theta, x)$ and the velocity $v(\theta)$ are given by

$$
v(\theta)=\rho c \frac{d}{2(a+b \theta)^{2}} \quad, \quad k(\theta, x)=\rho c \frac{1+d x}{(a+b \theta)^{2}}
$$

and $c, \rho$ and $l$ are the specific heat, the density and the latent heat of fusion of the medium respectively, all of them are assumed to be constant with positive parameters $a, d$ and real parameter $b$. This kind of nonlinear thermal conductivity or diffusion coefficients was considered in numerous papers, e.g. [3, 6, 7, 15, 20, 21, 25]. The nonlinear transport Eq.(1) arises in connection with unsaturated flow in heterogeneous porous media. If we set $d=0$ and $b=0$ in the free boundary problem $(1)-(5)$ then we retrieve the classical one-phase Lamé-Clapeyron-Stefan problem. The first explicit solution for the one-phase Stefan problem was given in [16]. Here $-q_{0} / \sqrt{t}$ denotes the prescribed flux on the boundary $x=0$ which is of the type imposed in [26]. We will determine which conditions on the parameters of the problem must be satisfied in order to have an instantaneous phasechange process.

In Section II we consider the free boundary problem (1) - (4) with the nonlinear heat coefficients (5) under the hypotheses $b>0$ and $a>b l / c$, or $b<0$. We follow $[19,24]$ and we improve [19] in the sense that the existence of the explicit solution of the problem $(1)-(5)$ is obtained through the unique solution of the Cauchy problem (55) - (56) in the spatial variable and the time $t$ is a parameter for $t \geqslant t_{0}>0$ where $t_{0}$ is an arbitrary positive time. This explicit solution can be obtained as a function of a parameter $\delta$ which is given as the unique solution of the transcendental Eq. (38). We also give an algorithm in order to compute the explicit solution for the temperature $\theta=\theta(x, t)$ and free boundary $x=s(t)$.

In Section III we consider the free boundary problem $(1),(3)-(4)$ with the nonlinear heat coefficients (5) and the temperature boundary condition (62) (with $\theta_{0}$ is a constant temperature given on the fixed boundary $x=0$ ) instead of the heat flux condition (2) 
under the hypotheses $a>0, d>0, b<0$ and $a+b \theta_{0}>0$. We improve [19] obtaining the explicit solution through the unique solution of the Cauchy problem (92) - (93) in the spatial variable and the time $t$ is a parameter for $t \geqslant t_{0}>0$ where $t_{0}$ is an arbitrary positive time. In this case, the explicit solution can be also obtained as a function of a parameter $\beta$ which is the unique solution of the Eq. (85). We also give an algorithm to compute the temperature $\theta=\theta(x, t)$ and free boundary $x=s(t)$. Other problems with nonlinear thermal coefficients in this subject are also given in $[4,5,18,22,23]$.

\section{Solution of the free boundary problem with heat flux condition on the fixed} face.

We consider the problem (1) - (4). Taking into account (5) we can put our problem as

$$
\begin{gathered}
\frac{\partial \theta}{\partial t}=\frac{\partial}{\partial x}\left(\frac{1+d x}{(a+b \theta)^{2}} \frac{\partial \theta}{\partial x}+\frac{d}{2 b(a+b \theta)}\right), 0<x<s(t), t>0 \\
\frac{1}{(a+b \theta(0, t))^{2}} \frac{\partial \theta}{\partial x}(0, t)=-\frac{q_{0}^{*}}{\sqrt{t}}, t>0 \\
\frac{1+d s(t)}{a^{2}} \frac{\partial \theta}{\partial x}(s(t), t)=-\alpha s(t), t>0 \\
\theta(s(t), t)=0, t>0, s(0)=0
\end{gathered}
$$

where $\alpha=\frac{l}{c}, q_{0}^{*}=\frac{q_{0}}{\rho c}$ and $a, d \in \mathbb{R}^{+}, b \in \mathbb{R}$.

If we define the transformations in the same way as in [20], [24]

$$
\left\{\begin{array}{l}
y=\frac{2}{d}\left[(1+d x)^{\frac{1}{2}}-1\right] \quad, \quad \bar{S}(t)=\frac{2}{d}\left[(1+d s(t))^{\frac{1}{2}}-1\right] \\
\bar{\theta}(y, t)=\theta(x, t)
\end{array}\right.
$$

we obtain the following free boundary problem

$$
\begin{gathered}
\frac{\partial \bar{\theta}}{\partial t}=\frac{\partial}{\partial y}\left(\frac{1}{(a+b \bar{\theta})^{2}} \frac{\partial \bar{\theta}}{\partial y}\right), 0<y<\bar{S}(t), t>0 \\
\frac{1}{(a+b \bar{\theta}(0, t))^{2}} \frac{\partial \bar{\theta}}{\partial y}(0, t)=-\frac{q_{0}^{*}}{\sqrt{t}}, t>0 \\
\frac{1}{a^{2}} \frac{\partial \bar{\theta}}{\partial y}(\bar{S}(t), t)=-\alpha \bar{S}(t), t>0
\end{gathered}
$$




$$
\bar{\theta}(\bar{S}(t), t)=0, t>0, \bar{S}(0)=0
$$

Then, we define the new transformation

$$
\left\{\begin{array}{l}
y^{*}=y^{*}(y, t)=\int_{S(t)}^{y}(a+b \bar{\theta}(\sigma, t)) d \sigma+(-\alpha b+a) \bar{S}(t) \\
\theta^{*}\left(y^{*}, t^{*}\right)=\frac{1}{a+b \bar{\theta}(y, t)}, t^{*}=t \\
S^{*}\left(t^{*}\right)=\left.y^{*}\right|_{y=\bar{S}(t)}=(-\alpha b+a) \bar{S}(t) .
\end{array}\right.
$$

In order to obtain an alternative expression for $y^{*}$ we compute

$$
\begin{aligned}
& \frac{\partial y^{*}}{\partial t}=-(a+b \bar{\theta}(\bar{S}(t), t)) \dot{\bar{S}}(t)+\int_{S(t)}^{y} b \frac{\partial \bar{\theta}}{\partial t}(\sigma, t) d \sigma+(-\alpha b+a) \dot{\bar{S}}(t)= \\
&=-\alpha b \dot{\bar{S}}(t)+\int_{S(t)}^{y} b \frac{\partial}{\partial \sigma}\left(\frac{1}{(a+b \bar{\theta}(\sigma, t))^{2}} \frac{\partial \bar{\theta}}{\partial \sigma}\right) d \sigma= \\
&=-\alpha b \dot{\bar{S}}(t)+b\left(\frac{1}{(a+b \bar{\theta}(y, t))^{2}} \frac{\partial \bar{\theta}}{\partial y}(y, t)-\frac{1}{a^{2}} \frac{\partial \bar{\theta}}{\partial y}(\bar{S}(t), t)\right)= \\
&=\frac{(a+b \bar{\theta}(y, t))^{2}}{\frac{\partial \bar{\theta}}{\partial y}(y, t)=} \\
&=\int_{0} \frac{\partial}{\partial \sigma}\left(\frac{b}{(a+b \bar{\theta}(\sigma, t))^{2}} \frac{\partial \bar{\theta}}{\partial \sigma}(\sigma, t)\right) d \sigma+\frac{b}{(a+b \bar{\theta}(0, t))^{2}} \frac{\partial \bar{\theta}}{\partial y}(0, t) \\
&=b \int_{0}^{y} \frac{\partial \bar{\theta}}{\partial t}(\sigma, t) d \sigma-\frac{b q_{0}^{*}}{\sqrt{t}},
\end{aligned}
$$

then the new expression for $y^{*}$ is given by

$$
\begin{gathered}
y^{*}(y, t)=\int_{0}^{t}\left(\int_{0}^{y} \frac{\partial}{\partial \sigma}(a+b \bar{\theta}(\sigma, \tau)) d \sigma-\frac{b q_{0}^{*}}{\sqrt{\tau}}\right) d \tau+\int_{0}^{y}(a+b \bar{\theta}(\sigma, 0)) d \sigma= \\
=\int_{0}^{y}(a+b \bar{\theta}(\sigma, t)) d \sigma-2 b q_{0}^{*} \sqrt{t} .
\end{gathered}
$$

Now, applying (10) and (15) the problem (6) - (9) is transformed in a Stefan-like problem with a convective boundary condition given by [28]

$$
\frac{\partial \theta^{*}}{\partial t^{*}}=\frac{\partial^{2} \theta^{*}}{\partial y^{* 2}},-2 b q_{0}^{*} \sqrt{t^{*}}<y^{*}<S^{*}\left(t^{*}\right), t^{*}>0
$$




$$
\begin{gathered}
\frac{\partial \theta^{*}}{\partial y^{*}}\left(-2 b q_{0}^{*} \sqrt{t^{*}}, t^{*}\right)=\frac{q_{0}^{*} b}{\sqrt{t^{*}}} \theta^{*}\left(-2 b q_{0}^{*} \sqrt{t^{*}}, t^{*}\right), t^{*}>0 \\
\frac{\partial \theta^{*}}{\partial y^{*}}\left(S^{*}\left(t^{*}\right), t^{*}\right)=\alpha^{*} \dot{S}^{*}\left(t^{*}\right), t^{*}>0 \\
\theta^{*}\left(S^{*}\left(t^{*}\right), t^{*}\right)=\theta_{f}^{*}, t^{*}>0, S^{*}(0)=0
\end{gathered}
$$

where

$$
\alpha^{*}=\frac{\alpha b}{a(-\alpha b+a)} \quad, \quad \theta_{f}^{*}=\frac{1}{a} .
$$

Then, if we introduce the similarity variable:

$$
\xi^{*}=\frac{y^{*}}{\sqrt{2 \gamma^{*} t^{*}}}
$$

where $\gamma^{*}$ is a dimensionless positive constant to be determined, and the solution is sought of the type

$$
\theta^{*}\left(y^{*}, t^{*}\right)=\Theta^{*}\left(\xi^{*}\right), \quad S^{*}\left(t^{*}\right)=\sqrt{2 \gamma^{*} t^{*}}
$$

then, we get that $(19)-(22)$ yields

$$
\begin{gathered}
\frac{d^{2} \Theta^{*}}{d \xi^{* 2}}+\gamma^{*} \xi^{*} \frac{d \Theta^{*}}{d \xi^{*}}=0,-b q_{0}^{*} \sqrt{\frac{2}{\gamma^{*}}}<\xi^{*}<1 \\
\frac{d \Theta^{*}}{d \xi^{*}}\left(-b q_{0}^{*} \sqrt{\frac{2}{\gamma^{*}}}\right)=\sqrt{2 \gamma^{*}} q_{0}^{*} b \Theta^{*}\left(-b q_{0}^{*} \sqrt{\frac{2}{\gamma^{*}}}\right) \\
\frac{d \Theta^{*}}{d \xi^{*}}(1)=\alpha^{*} \gamma^{*} \\
\Theta^{*}(1)=\theta_{f}^{*} .
\end{gathered}
$$

The solution of the differential equation (26) is given by

$$
\Theta^{*}\left(\xi^{*}\right)=A \operatorname{erf}\left(\sqrt{\frac{\gamma^{*}}{2}} \xi^{*}\right)+B
$$

where $A$ and $B$ are two unknown coefficients to be determined. From (28) and (29) we get

$$
A=\sqrt{\pi} \sqrt{\frac{\gamma^{*}}{2}} \alpha^{*} \exp \left(\frac{\gamma^{*}}{2}\right)
$$




$$
B=\frac{1}{a}-\sqrt{\pi} \sqrt{\frac{\gamma^{*}}{2}} \alpha^{*} \exp \left(\frac{\gamma^{*}}{2}\right) \operatorname{erf}\left(\sqrt{\frac{\gamma^{*}}{2}}\right)
$$

where

$$
\operatorname{erf}(x)=\frac{2}{\sqrt{\pi}} \int_{0}^{x} \exp \left(-u^{2}\right) d u
$$

The unknown constant $\gamma^{*}$ is determined by the remaining boundary condition (28) which yields the following equation

$$
\alpha^{*} \sqrt{\gamma^{*}}=\sqrt{\frac{2}{\pi}} \frac{\theta_{f}^{*}}{g\left(b q_{0}^{*}, \frac{1}{\sqrt{\pi}}\right)+\operatorname{erf}\left(\sqrt{\frac{\gamma^{*}}{2}}\right)} \exp \left(-\frac{\gamma^{*}}{2}\right), \gamma^{*}>0
$$

where

$$
\begin{aligned}
& g(x, p)=\operatorname{erf}(x)+p R(x), x>0, p \in \mathbb{R} \\
& R(x)=\frac{\exp \left(-x^{2}\right)}{x}=\frac{1}{P(x)}, P(x)=x \exp \left(x^{2}\right), x>0 \\
& E(x)=x \exp \left(x^{2}\right) \operatorname{erf}(x)=P(x) \operatorname{erf}(x), x>0 .
\end{aligned}
$$

By defining the new unknown

$$
\delta=\sqrt{\frac{\gamma^{*}}{2}}
$$

the equation (34) is equivalent to the equation

$$
g\left(b q_{0}^{*}, \frac{1}{\sqrt{\pi}}\right) P(\delta)=\frac{\frac{a c}{b l}-1}{\sqrt{\pi}}-E(\delta), \quad \delta>0,
$$

that is

$$
g\left(\delta, \frac{1}{\sqrt{\pi}}\left(1-\frac{a}{\alpha b}\right)\right)=-g\left(b q_{0}^{*}, \frac{1}{\sqrt{\pi}}\right) \quad, \quad \delta>0
$$

Theorem 1.- If $b>0$ and $a>\frac{b l}{c}$, or $b<0$, then the free boundary problem (1) - (4) has a unique solution of the similarity type which is given by

$$
\begin{aligned}
& \theta(\xi)=\frac{1}{b}\left[\frac{1}{A \operatorname{erf}\left(\sqrt{\frac{\gamma^{*}}{2}} \xi^{*}\right)+B}-a\right] \\
& \xi=\frac{y}{\sqrt{2 \gamma t}}=\frac{\frac{2}{d}\left[(1+d x)^{\frac{1}{2}}-1\right]}{\sqrt{2 \gamma t}} \\
& s(t)=\frac{1}{d}\left[\left(1+\frac{d}{2} \sqrt{2 \gamma t}\right)^{2}-1\right]
\end{aligned}
$$


where

$$
\xi=(-\alpha b+a) \int_{-\sqrt{\frac{2}{\gamma^{*}}} b q_{0}^{*}}^{\xi^{*}}\left[A \operatorname{erf}\left(\sqrt{\frac{\gamma^{*}}{2}} \sigma\right)+B\right] d \sigma
$$

and $A, B$ and $\gamma$ are given by (31), (32) and (43) respectively.

Proof. First, we have to study the existence and uniqueness of the equation (34) or (38). It's easy to see that $g(0, p)=-\infty, g(+\infty, p)=1$ and $\frac{\partial g}{\partial x}(x, p)>0, \forall x>0, \forall p<0$ [4]. Therefore if we have $b>0$ and

$$
1-\frac{a}{\alpha b}<0\left(\text { that is } a>\alpha b=\frac{b l}{c}\right)
$$

and taking into account that $g\left(b q_{0}^{*}, \frac{1}{\sqrt{\pi}}\right)>1$, the equation (38) has a unique solution $\delta>0$. From (25) we obtain the expression of $S(t)$ given by

$$
S(t)=\sqrt{2 \gamma t}
$$

where $\gamma$ is given by

$$
\gamma=\frac{\gamma^{*}}{(-\alpha b+a)^{2}}
$$

From $(24),(25)$ and (30) we can obtain the parametric solution of the problem (1) - (4) given by (39) and (40). Note that $\left.\xi^{*}\right|_{\xi=0}=\frac{-2 b q_{0}^{*} \sqrt{t^{*}}}{\sqrt{2 \gamma^{*} t^{*}}}=-\sqrt{\frac{2}{\gamma^{*}}} b q_{0}^{*}=-\frac{b q_{0}^{*}}{\delta}$.

If we have now $b<0$ then the equation (38) for $\delta$ is given by

$$
g\left(\delta, \alpha_{0}\right)=-g\left(-b q_{0}^{*}, \frac{1}{\sqrt{\pi}}\right) \quad, \quad \delta>0
$$

which has a unique solution $\delta>0$ taking into account that

$$
\alpha_{0}=\frac{1}{\sqrt{\pi}}\left(1-\frac{a}{\alpha b}\right)>0, \quad g\left(-b q_{0}^{*}, \frac{1}{\sqrt{\pi}}\right)>1, \quad \forall b<0 .
$$

Remark 1. There does not exist any solution to the free boundary problem (1) - (4) if $b>0$ and $0<a<\frac{b l}{c}$ because the non existence of a real solution of the Eq.(38). have:

In order to obtain the explicit solution for the cases $b>0$ and $a>\frac{b l}{c}$, or $b<0$ we

(i) There exists a unique $\delta>0$ solution of the Eq. (38).

(ii) We have

$$
\gamma^{*}=2 \delta^{2}, A=\alpha^{*} \sqrt{\pi} P(\delta), B=\theta_{f}^{*}-\alpha^{*} \sqrt{\pi} E(\delta)
$$

where

$$
\alpha^{*}=\frac{\alpha b}{a(a-\alpha b)}, \theta_{f}^{*}=\frac{1}{a}, \alpha=l / c .
$$


(iii) We have

$$
\theta^{*}\left(y^{*}, t^{*}\right)=\Theta^{*}\left(\xi^{*}\right)=A \operatorname{erf}\left(\sqrt{\frac{\gamma^{*}}{2}} \xi^{*}\right)+B=B+A \operatorname{erf}\left(\delta \xi^{*}\right)
$$

with $\xi^{*}=\frac{y^{*}}{2 \delta \sqrt{t}}$ and

$$
S^{*}(t)=\sqrt{2 \gamma^{*} t}=2 \delta \sqrt{t}
$$

(iv) We have

$$
y^{*}(y, t)=\int_{0}^{y}(a+b \bar{\theta}(\sigma, t)) d \sigma-2 b q_{0}^{*} \sqrt{t}, q_{0}^{*}=q_{0} / \rho c .
$$

(v) We have

$$
\begin{gathered}
\bar{S}(t)=\frac{S^{*}(t)}{a-\alpha b}=\frac{2 \delta}{a-\alpha b} \sqrt{t} \\
\frac{1}{a+b \bar{\theta}(y, t)}=\theta^{*}\left(y^{*}, t\right)=\Theta^{*}\left(\xi^{*}\right)=A \operatorname{erf}\left(\frac{y^{*}}{2 \sqrt{t}}\right)+B= \\
=B+A \operatorname{erf}\left[\frac{1}{2 \sqrt{t}} \int_{0}^{y}(a+b \bar{\theta}(\sigma, t)) d \sigma-b q_{0}^{*}\right]
\end{gathered}
$$

which is an integral equation for $\bar{\theta}=\bar{\theta}(y, t)$ where $t>0$ is a parameter.

(vi) The free boundary $s=s(t)$ is given by:

$$
\begin{aligned}
s(t) & =\frac{1}{d}\left[\left(1+\frac{d}{2} \bar{S}(t)\right)^{2}-1\right]=\frac{1}{d}\left[\left(1+\frac{d \delta}{a-\alpha b} \sqrt{t}\right)^{2}-1\right]= \\
& =\frac{2 \delta}{a-\alpha b} \sqrt{t}+\frac{d \delta^{2}}{(a-\alpha b)^{2}} t=\frac{\delta}{a-\alpha b}\left[2 \sqrt{t}+\frac{d \delta}{a-\alpha b} t\right]
\end{aligned}
$$

(vii) If we define

$$
Y(y, t)=-b q_{0}^{*}=\frac{1}{2 \sqrt{t}} \int_{0}^{y}(a+b \bar{\theta}(\sigma, t)) d \sigma
$$

then we obtain

$$
\frac{\partial Y}{\partial y}(y, t)=\frac{a+b \bar{\theta}(y, t)}{2 \sqrt{t}}
$$

that is $Y=Y(y, t)$ satisfies the following Cauchy problem in variable $y$ :

$$
\begin{gathered}
\frac{\partial Y}{\partial y}(y, t)=\frac{1}{2 \sqrt{t}}\left(\frac{1}{B+A \operatorname{erf}(Y(y, t))}\right), 0<y<\bar{S}(t), t>0 \\
Y(0, t)=-b q_{0}^{*},
\end{gathered}
$$

where $t>0$ is a parameter. 
(viii) The temperature $\bar{\theta}=\bar{\theta}(y, t)$ is given by:

$$
\bar{\theta}(y, t)=\frac{1}{b}\left[\frac{1}{B+A \operatorname{erf}(Y(y, t))}-a\right], 0<y<\bar{S}(t), t>0
$$

as a function of $Y$.

$(i x)$ The temperature $\theta=\theta(x, t)$ is given by:

$$
\begin{gathered}
\theta(x, t)=\bar{\theta}\left(\frac{2}{d}(\sqrt{1+d x}-1), t\right)= \\
=\frac{1}{b}\left[\frac{1}{B+A \operatorname{erf}\left(Y\left(\frac{2}{d}(\sqrt{1+d x}-1), t\right)\right)}-a\right], 0<x<s(t), t>0
\end{gathered}
$$

as a function of $Y$ where $s(t)$ as defined in (52).

Theorem 2 Let us consider the hypothesis $b>0$ and $a>b l / c$, or $b<0$. Let $\delta>0$ be the unique solution of the Eq. (38) and $A$ and $B$ the coefficients defined by (31) and (32) respectively or by (46).

(i) There exists a unique solution $Y=Y(y, t)$ of the Cauchy problem (55) - (56) for all $t \geqslant t_{0}>0$ where $t_{0}$ is an arbitrary positive time.

(ii) There exists a unique solution $\theta=\theta(x, t)$ and $s=s(t)$ given by (58) and (52) respectively of the free boundary problem (1) - (4) for $t \geqslant t_{0}>0$ where $t_{0}$ is an arbitrary positive time.

\section{Proof:}

It is sufficient to prove that the Cauchy problem has a unique solution for $t \geqslant t_{0}>0$. The ordinary differential equation, with parameter $t>0$, can be written as

$$
\left.\frac{\partial Y}{\partial y}(y, t)=G(y, t, Y(y, t))\right)
$$

where

$$
G(y, t, Y)=\frac{1}{2 \sqrt{t}}\left(\frac{1}{B+A \operatorname{erf}(Y)}\right)
$$

satisfies the condition

$$
\left|\frac{\partial G}{\partial Y}(y, t, Y)\right| \leq \text { Const } \quad, \quad \forall t \geqslant t_{0}>0
$$

with $t_{0}>0$ an arbitrary positive time.

Remark 2. The existence of a solution for $t \geqslant t_{0}>0$ with $t_{0}$ an arbitrary positive time for the cases $b>0$ and $a>b l / c$, or $b<0$ is similar to the one obtained in the free boundary problem studied in [20].

\section{Remark 3:}

The particular case $b>0$ and $a=b l / c$ can not be studied through a similar method developed for the case $b>0$ and $a>b l / c$ because the transformation (15) is not useful due to the definition of the free boundary $S^{*}\left(t^{*}\right)$ as a function of the $\bar{S}(t)$.

\section{Remark 4:}

For the cases $b>0$ and $a>b l / c$, or $b<0$ we can obtain the explicit solution $\theta=\theta(x, t)$ and $s=s(t)$ of the free boundary problem (1) - (4) by the following process: 
(i) Compute $\delta>0$ as the unique solution of the Eq. (38).

(ii) Compute

$$
A=\alpha^{*} \sqrt{\pi} P(\delta), \quad B=\theta_{f}^{*}-\alpha^{*} \sqrt{\pi} E(\delta)
$$

where $\alpha^{*}, \theta_{f}^{*}$ and $\alpha$ are defined in (47).

(iii) Fix $t_{0}$ as an arbitrary positive time and compute $Y=Y(y, t)$ as the unique solution of the Cauchy problem (55) $-(56)$ for $t \geqslant t_{0}>0$.

(iv) Compute the free boundary $s=s(t)$ by the explicit expression (52)

$(v)$ Compute the temperature $\theta=\theta(x, t)$ by the explicit expression (58).

III. Solution of the free boundary problem with temperature boundary condition on the fixed face.

Now, we consider the problem (1) - (4) with $a, d \in \mathbb{R}^{+}$and $b<0$ but the heat flux boundary condition (2) will be replaced by the following temperature boundary condition $\left(\theta_{0}>0\right)$ given by

$$
\theta(0, t)=\theta_{0}, t>0, \text { with } a+b \theta_{0}>0
$$

We can define the same transformations (10) and (15) as were done for the previous Section but now we get

$$
\begin{aligned}
\frac{\partial y^{*}}{\partial t} & =b \int_{S(t)}^{y} \frac{\partial \bar{\theta}}{\partial t}(\sigma, t) d \sigma+\frac{b}{(a+b \bar{\theta}(0, t))^{2}} \frac{\partial \bar{\theta}}{\partial y}(0, t)= \\
& =b \int_{0}^{y} \frac{\partial \bar{\theta}}{\partial t}(\sigma, t) d \sigma+\frac{b}{\left(a+b \theta_{0}\right)^{2}} \frac{\partial \bar{\theta}}{\partial y}(0, t) .
\end{aligned}
$$

Then

$$
\begin{gathered}
y^{*}(y, t)=\int_{0}^{t}\left(\int_{0}^{y} \frac{\partial}{\partial \tau}(a+b \bar{\theta}(\sigma, \tau)) d \sigma+\frac{b}{\left(a+b \theta_{0}\right)^{2}} \frac{\partial \bar{\theta}}{\partial y}(0, \tau)\right) d \tau+\int_{0}^{y}(a+b \bar{\theta}(\sigma, 0)) d \sigma= \\
=\int_{0}^{y}(a+b \bar{\theta}(\sigma, t)) d \sigma+\frac{b}{\left(a+b \theta_{0}\right)^{2}} \int_{0}^{t} \frac{\partial \bar{\theta}}{\partial y}(0, \tau) d \tau
\end{gathered}
$$

Therefore our free boundary problem becomes (21) - (22) and

$$
\begin{gathered}
\frac{\partial \theta^{*}}{\partial t}=\frac{\partial^{2} \theta^{*}}{\partial y^{*}}, b \theta_{0}^{* 2} \int_{0}^{t^{*}} \frac{\partial \bar{\theta}}{\partial y}(0, \tau) d \tau<y^{*}<S^{*}\left(t^{*}\right), t^{*}>0 \\
\theta^{*}\left(b \theta_{0}^{* 2} \int_{0}^{t^{*}} \frac{\partial \bar{\theta}}{\partial y}(0, \tau) d \tau, t^{*}\right)=\theta_{0}^{*}
\end{gathered}
$$


where $\theta_{f}^{*}$ and $\alpha^{*}$ are given by (23) and

$$
\theta_{0}^{*}=\frac{1}{a+b \bar{\theta}(0, t)}=\frac{1}{a+b \theta(0, t)}=\frac{1}{a+b \theta_{0}}
$$

It is easy to see that we have a classical Stefan problem so that the free boundary must be of the type

$$
S^{*}\left(t^{*}\right)=\sqrt{2 \gamma^{*} t^{*}} \quad\left(\bar{S}(t)=\sqrt{2 \gamma t}, \gamma^{*}=\gamma(a-\alpha b)^{2}\right)
$$

where $\gamma^{*}$ (i.e. $\gamma$ ) is a dimensionless constant to be determined.

If we introduce the similarity variable (24) and we propose the solution of the type (25) then the problem $(21)-(22)$ and $(64)-(65)$ yields $(28),(29)$ and

$$
\begin{gathered}
\frac{d^{2} \Theta^{*}}{d \xi^{* 2}}+\gamma^{*} \xi^{*} \frac{d \Theta^{*}}{d \xi^{*}}=0, \frac{b \theta_{0}^{* 2}}{\sqrt{2 \gamma^{*} t^{*}}} \int_{0}^{t^{*}} \frac{\partial \bar{\theta}}{\partial y}(0, \tau) d \tau<\xi^{*}<1, t^{*}>0 \\
\Theta^{*}\left(\frac{b \theta_{0}^{* 2}}{\sqrt{2 \gamma^{*} t^{*}}} \int_{0}^{t^{*}} \frac{\partial \bar{\theta}}{\partial y}(0, \tau) d \tau\right)=\theta_{0}^{*}, t^{*}>0
\end{gathered}
$$

From (69) we must necessarily have that there exists a constant $\xi_{0}^{*}$ such that

$$
\int_{0}^{t^{*}} \frac{\partial \bar{\theta}}{\partial y}(0, \tau) d \tau=\xi_{0}^{*} \frac{1}{b \theta_{0}^{* 2}} \sqrt{2 \gamma^{*} t^{*}}
$$

Therefore (68) and (69) can be written as

$$
\begin{gathered}
\frac{d^{2} \Theta^{*}}{d \xi^{* 2}}+\gamma^{*} \xi^{*} \frac{d \Theta^{*}}{d \xi^{*}}=0, \xi_{0}^{*}<\xi^{*}<1 \\
\Theta^{*}\left(\xi_{0}^{*}\right)=\theta_{0}^{*} .
\end{gathered}
$$

The solution of the problem $(28),(29),(71)$ and (72) is given by

$$
\Theta^{*}\left(\xi^{*}\right)=A^{\prime} \operatorname{erf}\left(\sqrt{\frac{\gamma^{*}}{2}} \xi^{*}\right)+B^{\prime} \quad, \quad \xi_{0}^{*}<\xi^{*}<1
$$

where the unknown coefficients $\xi_{0}^{*}, A^{\prime}, B^{\prime}$ and $\gamma^{*}$ must satisfy the following equations

$$
\begin{gathered}
A^{\prime} \operatorname{erf}\left(\xi_{0}^{*} \sqrt{\frac{\gamma^{*}}{2}}\right)+B^{\prime}=\theta_{0}^{*} \quad, \quad \sqrt{\frac{2}{\pi \gamma^{*}}} \exp \left(-\frac{\gamma^{*}}{2}\right)=\frac{\alpha^{*}}{A^{\prime}}, \\
A^{\prime} \operatorname{erf}\left(\sqrt{\frac{\gamma^{*}}{2}}\right)+B^{\prime}=\theta_{f}^{*} \text { and } \theta_{0}^{*} \sqrt{\frac{\gamma^{*}}{2}} \xi_{0}^{*}=-\frac{A^{\prime}}{\sqrt{\pi}} \exp \left(-\frac{\gamma^{*}}{2} \xi_{0}^{* 2}\right)
\end{gathered}
$$


If we define

$$
\beta=\sqrt{\frac{\gamma^{*}}{2}}, z=\xi_{0}^{*} \sqrt{\frac{\gamma^{*}}{2}} \quad(\beta>z)
$$

we have to solve the following system of equations:

$$
\begin{gathered}
A^{\prime} \operatorname{erf}(z)+B^{\prime}=\theta_{0}^{*}, \quad A^{\prime} \operatorname{erf}(\beta)+B^{\prime}=\theta_{f}^{*} \\
\frac{\exp \left(-\beta^{2}\right)}{\beta}=\frac{\alpha^{*}}{A^{\prime}} \sqrt{\pi}, \quad \theta_{0}^{*} z=-\frac{A^{\prime}}{\sqrt{\pi}} \exp \left(-z^{2}\right)
\end{gathered}
$$

From (77) we get

$$
\begin{gathered}
A^{\prime}=\frac{\theta_{f}^{*}-\theta_{0}^{*}}{\operatorname{erf}(\beta)-\operatorname{erf}(z)} \\
B^{\prime}=\frac{\theta_{0}^{*} \operatorname{erf}(\beta)-\theta_{f}^{*} \operatorname{erf}(z)}{\operatorname{erf}(\beta)-\operatorname{erf}(z)}
\end{gathered}
$$

and from (78) we obtain

$$
\begin{aligned}
& \frac{\exp \left(-\beta^{2}\right)}{\beta}=\delta_{1}[\operatorname{erf}(\beta)-\operatorname{erf}(z)] \\
& \frac{\exp \left(-z^{2}\right)}{z}=\delta_{2}[\operatorname{erf}(\beta)-\operatorname{erf}(z)]
\end{aligned}
$$

with

$$
\delta_{1}=\frac{\alpha^{*} \sqrt{\pi}}{\theta_{f}^{*}-\theta_{0}^{*}}=\frac{\alpha \sqrt{\pi}\left(a+b \theta_{0}\right)}{(a-\alpha b) \theta_{0}} \quad, \quad \delta_{2}=-\frac{\sqrt{\pi} a}{b \theta_{0}} .
$$

Taking into account that $\xi_{0}^{*}<1, b<0$ and $a+b \theta_{0}>0$, we have $[\operatorname{erf}(\beta)-\operatorname{erf}(z)]>0$, $\delta_{1}>0$ and $\delta_{2}>0$.

Taking into account the properties of the real function $g=g(x, p)$, defined in (35), for $p<0$, from (81) we have

$$
z=\operatorname{erf}^{-1}\left[\operatorname{erf}(\beta)-\frac{1}{\delta_{1}} \frac{\exp \left(-\beta^{2}\right)}{\beta}\right]
$$

if $\beta>x_{0}$ where $x_{0}>0$ is the unique positive zero of $g\left(x,-\frac{1}{\delta_{1}}\right)=0$ which is given by $x_{0}=E^{-1}\left(1 / \delta_{1}\right)$.

Moreover, for $\beta$ we have the following equation:

$$
\frac{\delta_{2}}{\delta_{1}} \frac{F(\beta)}{\beta \exp \left(\beta^{2}\right)}=1, \beta>x_{0}=E^{-1}\left(1 / \delta_{1}\right)
$$

where the real function $F$ is defined by

$$
F(x)=\exp \left[\left(\operatorname{erf}^{-1}\left(g\left(x, \frac{-1}{\delta_{1}}\right)\right)\right)^{2}\right] \operatorname{erf}^{-1}\left(g\left(x, \frac{-1}{\delta_{1}}\right)\right), x>0 .
$$


If we define the real function:

$$
G(x)=\frac{\delta_{2}}{\delta_{1}} \frac{F(x)}{x \exp \left(x^{2}\right)}, x>0
$$

and following [20] we have the following properties:

$$
G\left(x_{0}^{+}\right)=0, \quad \lim _{x \rightarrow+\infty} G(x)=\frac{\delta_{2}}{\delta_{1}+\sqrt{\pi}} .
$$

Then there exists a unique solution $\beta>x_{0}$ of the Eq. (85) if and only if $\frac{\delta_{2}}{\delta_{1}+\sqrt{\pi}}>1$ if and only if $a+b \theta_{0}>0$ which is our hypothesis.

Moreover, we have

$$
\bar{S}(t)=\frac{2 \beta}{a-\alpha b} \sqrt{t}
$$

and for $0<y<\bar{S}(t), t>0$, we obtain

$$
\begin{aligned}
\frac{1}{a+b \bar{\theta}(y, t)} & =\theta^{*}\left(y^{*}, t\right)=\Theta^{*}\left(\xi^{*}\right)=A^{\prime} \operatorname{erf}\left(\beta \xi^{*}\right)+B^{\prime}= \\
& =B^{\prime}+A^{\prime} \operatorname{erf}\left(\frac{y^{*}}{2 \sqrt{t}}\right)= \\
& =B^{\prime}+A^{\prime} \operatorname{erf}\left[z+\frac{1}{2 \sqrt{t}} \int_{0}^{y}(a+b \bar{\theta}(\sigma, t)) d \sigma\right]
\end{aligned}
$$

which represents an integral equation for $\bar{\theta}$ in variable y with $t$ a parameter.

In order to solve this integral equation we define

$$
Y(y, t)=z+\frac{1}{2 \sqrt{t}} \int_{0}^{y}(a+b \bar{\theta}(\sigma, t)) d \sigma,
$$

which must satisfy the following Cauchy problem

$$
\begin{gathered}
\frac{\partial Y}{\partial y}(y, t)=\frac{1}{2 \sqrt{t}}\left(\frac{1}{B^{\prime}+A^{\prime} \operatorname{erf}(Y(y, t))}\right), 0<y<\bar{S}(t), t>0 \\
Y(0, t)=z
\end{gathered}
$$

Therefore we obtain the following theorem.

Theorem 3. Let us consider the hypothesis $a>0, d>0, b<0$ and $a+b \theta_{0}>0$. Let $\beta>0$ be the unique solution of the Eq. (85), and $A^{\prime}$ and $B^{\prime}$ the coefficients defined by (79) and (80) respectively.

( $i$ ) There exists a unique solution $Y=Y(y, t)$ of the Cauchy problem (92) - (93) for all $t \geqslant t_{0}>0$ where $t_{0}>0$ is an arbitrary positive time. 
(ii) There exists a unique solution $\theta=\theta(x, t)$ and $s=s(t)$ given by (95) and (96) respectively of the free boundary problem (1), (3) - (4) and (62).

\section{Proof.}

(i) The Cauchy problem (92) - (93) has a unique solution $Y=Y(y, t)$ for all $t \geqslant t_{0}>0$ with $t_{0}>0$ is an arbitrary positive time following a similar method given in Theorem 2 .

(ii) From (90) and (91) we get

$$
\bar{\theta}(y, t)=\frac{1}{b}\left[\frac{1}{B^{\prime}+A^{\prime} \operatorname{erf}(Y(y, t))}-a\right]
$$

that is

$$
\begin{aligned}
& \theta(x, t)=\bar{\theta}(y, t)=\bar{\theta}\left(\frac{2}{d}(\sqrt{1+d x}-1), t\right)= \\
& =\frac{1}{b}\left[\frac{1}{B^{\prime}+A^{\prime} \operatorname{erf}\left(Y\left(\frac{2}{d}(\sqrt{1+d x}-1), t\right)\right)}-a\right]
\end{aligned}
$$

and

$$
\begin{gathered}
s(t)=\frac{1}{d}\left[\left(1+\frac{d}{2} \bar{S}(t)\right)^{2}-1\right]=\frac{1}{d}\left[\left(1+\frac{d \beta}{a-\alpha b} \sqrt{t}\right)^{2}-1\right]= \\
=\frac{\beta}{a-\alpha b}\left[2 \sqrt{t}+\frac{d \beta}{a-\alpha b} t\right]
\end{gathered}
$$

Remark 5 For the case $a>0, d>0, b<0$ and $a+b \theta_{0}>0$ we can obtain the explicit solution $\theta=\theta(x, t)$ and $s=s(t)$ of the free boundary (1), (3) - (4) and (62) by the following process:

(i) Compute the positive parameters $\delta_{1}$ and $\delta_{2}$ given by (83).

(ii) Compute $\beta>0$ as the unique solution of the Eq. (85)

(iii) Compute the coefficients

$$
\begin{aligned}
z & =\operatorname{erf}^{-1}\left[\operatorname{erf}(\beta)-\frac{1}{\delta_{1}} \frac{\exp \left(-\beta^{2}\right)}{\beta}\right] \\
\gamma^{*} & =2 \beta^{2}, \gamma=\frac{\gamma^{*}}{(a-\alpha b)^{2}}=2\left(\frac{\beta}{a-\alpha b}\right)^{2} \\
\xi_{0}^{*} & =\frac{z}{\beta}
\end{aligned}
$$

(iv) Compute the coefficients $A^{\prime}<0$ and $B^{\prime}>0$ given by (79) and (80) respectively.

(v) Fix $t_{0}$ as an arbitrary positive time and compute $Y=Y(y, t)$ as the unique solution of the Cauchy problem (92) - (93).

(vi) Compute the free boundary $s=s(t)$ by the explicit expression (96)

(vi) Compute the temperature $\theta=\theta(x, t)$ by the explicit expression (95).

Acknowledgments. This paper has been partially sponsored by the project PIP $\mathrm{N}^{o}$ 5379 from CONICET, Rosario (Argentina) and by "Fondo de Ayuda a la Investigación" through Universidad Austral, Rosario (Argentina). 


\section{References}

[1] V. Alexiades and A.D. Solomon, Mathematical modeling of melting and freezing processes, Hemisphere - Taylor \& Francis, Washington (1983).

[2] I. Athanasopoulos, G. Makrakis and J.F. Rodrigues (Eds.), Free Boundary Problems: Theory and Applications, CRC Press, Boca Raton (1999).

[3] G. Bluman, S. Kumei, On the remarkable nonlinear diffusion equation, J. Math Phys. 21, 1019-1023 (1980).

[4] A. C. Briozzo, M. F. Natale and D. A. Tarzia, Determination of unknown thermal coefficients for Storm's-type materials through a phase-change process, Int. J. NonLinear Mech. 34, 324-340 (1999).

[5] A. C. Briozzo and D. A. Tarzia, " An explicit solution for an instantaneous two-phase Stefan problem with nonlinear thermal coefficients", IMA J. of Applied Mathematics, 67, 249-261 (2002).

[6] P. Broadbridge, Non-integrability of non-linear diffusion-convection equations in two spatial dimensions, J. Phys. A: Math. Gen 19, 1245-1257 (1986).

[7] P. Broadbridge, Integrable forms of the one-dimensional flow equation for unsaturated heterogeneous porous media, J. Math. Phys. 29, 622-627 (1988).

[8] J. R. Cannon, The one-dimensional heat equation, Addison - Wesley, Menlo Park (1984).

[9] H. S. Carslaw and J. C. Jaeger, Conduction of heat in solids, Oxford University Press, London (1959).

[10] J. M. Chadam and H. Rasmussen H (Eds.), Free boundary problems involving solids. Pitman Research Notes in Mathematics Series 281, Longman, Essex (1993).

[11] J. Crank J, Free and moving boundary problems, Clarendon Press, Oxford (1984).

[12] J. I. Diaz, M. A. Herrero, A. Liñan and J. L. Vazquez (Eds.), Free boundary problems: theory and applications, Pitman Research Notes in Mathematics Series 323, Longman, Essex (1995).

[13] A. Fasano and M. Primicerio (Eds.), Nonlinear diffusion problems, Lecture Notes in Math. N.1224, Springer Verlag, Berlin (1986).

[14] N. Kenmochi (Ed.), Free Boundary Problems: Theory and Applications, I,II, Gakuto International Series: Mathematical Sciences and Applications, Vol. 13, 14, Gakkotosho, Tokyo (2000).

[15] J. H. Knight, J. R. Philip, Exact solutions in nonlinear diffusion, J. Engrg. Math., 8, 219-227 (1974).

[16] G. Lamé and B. P. Clapeyron, Memoire sur la solidification par refroidissement d'un globe liquide, Annales Chimie Physique 47, 250-256 (1831). 
[17] V. J. Lunardini, Heat transfer with freezing and thawing, Elsevier, Amsterdam (1991).

[18] M. F. Natale and D. A. Tarzia, Explicit solutions to the two-phase Stefan problem for Storm-type materials, J. Phys. A: Math. Gen 33, 395-404 (2000).

[19] M. F. Natale and D. A. Tarzia, Explicit solutions to the one-phase Stefan problem with temperature-dependent thermal conductivity and a convective term, Int. J. Engng. Sci., 41 (2003), 1685-1698.

[20] M. F. Natale and D. A. Tarzia, Explicit solutions for a one-phase Stefan problem with temperature-dependent thermal conductivity, Bolletino Un. Mat. Italiana (8) 9$B$ (2006), 79-99.

[21] R. Philip, General method of exact solution of the concentration-dependent diffusion equation, Australian J. Physics, 13, 1-12 (1960).

[22] C. Rogers, Application of a reciprocal transformation to a two-phase Stefan problem, J. Phys. A: Math. Gen 18, 105-109 (1985).

[23] C. Rogers, On a class of moving boundary problems in non-linear heat condition: Application of a Bäcklund transformation, Int. J. Non-Linear Mech. 21, 249-256 (1986).

[24] C. Rogers and P. Broadbridge, On a nonlinear moving boundary problem with heterogeneity: application of reciprocal transformation, Journal of Applied Mathematics and Physics (ZAMP) 39, 122-129 (1988).

[25] G. C. Sander, I. F. Cunning, W. L. Hogarth and J. Y. Parlange, Exact solution for nonlinear nonhysteretic redistribution in vertical soli of finite depth, Water Resources Research 27, 1529-1536 (1991).

[26] D. A. Tarzia, An inequality for the coefficient $\sigma$ of the free boundary $s(t)=2 \sigma \sqrt{t}$ of the Neumann solution for the two-phase Stefan problem, Quart. Appl. Math 39, 491-497 (1981).

[27] D. A. Tarzia, A bibliography on moving - free boundary problems for the heat-diffusion equation. The Stefan and related problems, MAT-Serie A \#2 (2000) (with 5869 titles on the subject, 300 pages). See www.austral.edu.ar/MAT-SerieA/2(2000)/ or http://www.austral.edu.ar/fce/archivos/mat/Tarzia-MAT-SerieA-2(2000).pdf

[28] D. A. Tarzia, "An explicit solution for a wo-phase unidimensional Stefan problem with a convective boundary condition at the fixed face", MAT-SerieA, 8 (2004), $21-27$. 